Supporting Information

\title{
Phase Separation and d Electronic Orbitals on Cyclic Degradation in Li-Mn-O Compounds: First-Principles Multiscale Modeling and Experimental Observations
}

Duho Kim, $,{ }^{\dagger}, \|$ Jin-Myoung Lim, ${ }^{\dagger,} \|$ Min-Sik Park, ${ }^{\ddagger}$ Kyeongjae Cho, ${ }^{\S}$ and Maenghyo Cho ${ }^{*}, \dagger$

${ }^{\dagger}$ Department of Mechanical and Aerospace Engineering, Seoul National University, Gwanak-ro

1, Gwanak-gu, Seoul 08826, Republic of Korea

${ }^{\ddagger}$ Department of Advanced Materials Engineering for Information and Electronics, Kyung Hee University, 1732 Deogyeong-daero, Giheung-gu, Yongin 17104, Republic of Korea

$\S^{\S}$ Department of Materials Science and Engineering and Department of Physics, University of Texas at Dallas, Richardson, TX 75080, USA

"D. Kim and J.-M. Lim contributed equally to this work.

*E-mail:mhcho@snu.ac.kr (M. Cho) 
(a)

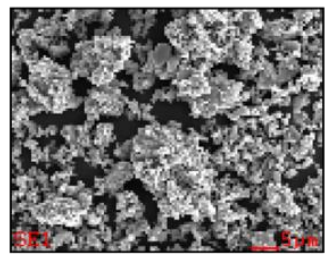

(d)

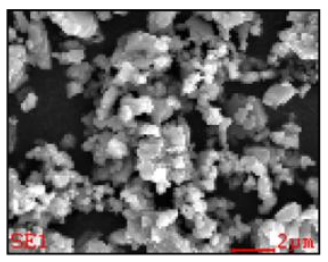

(h)

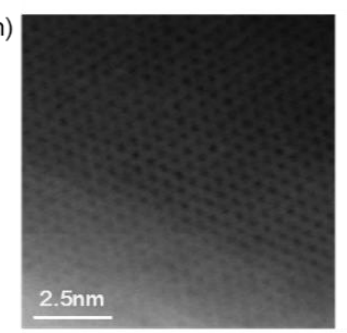

(k)

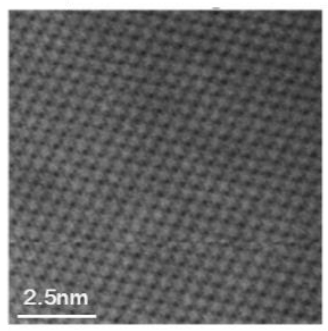

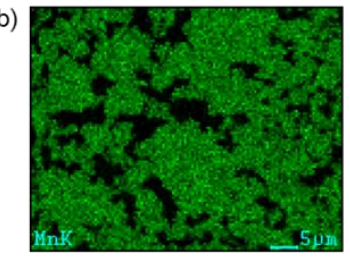

(e)

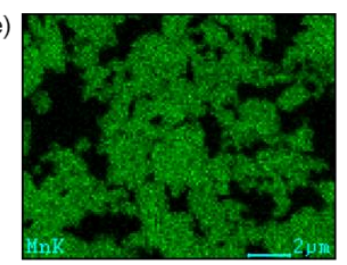

$\mathrm{Mn} \mathrm{Kal}$

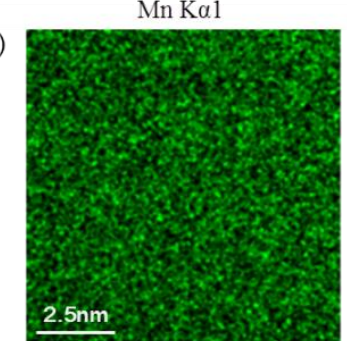

$\mathrm{Mn} \mathrm{K} \alpha 1$

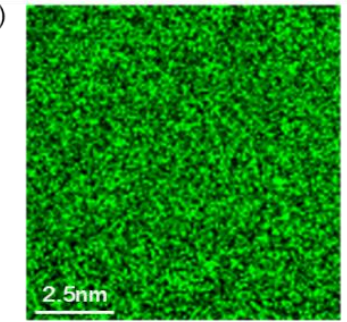

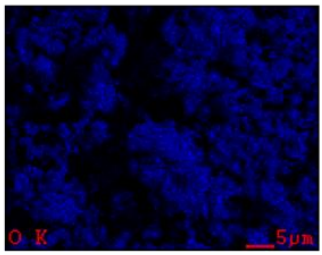

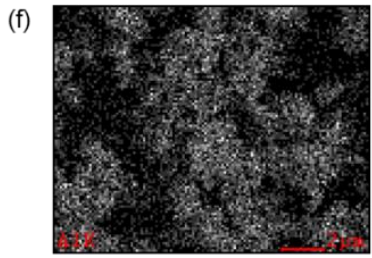

$\mathrm{OK} \alpha 1$

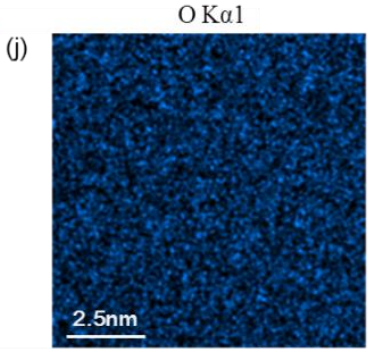

$\mathrm{Al} \mathrm{K} \alpha 1$

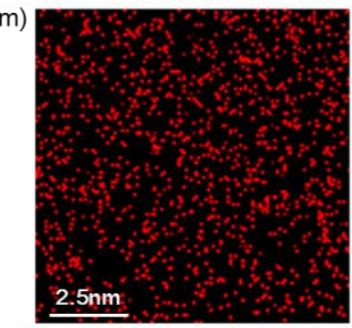

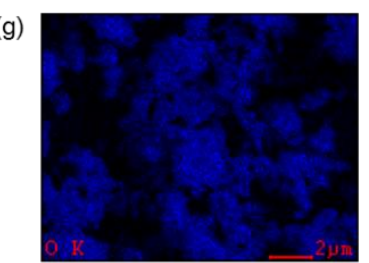

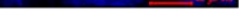



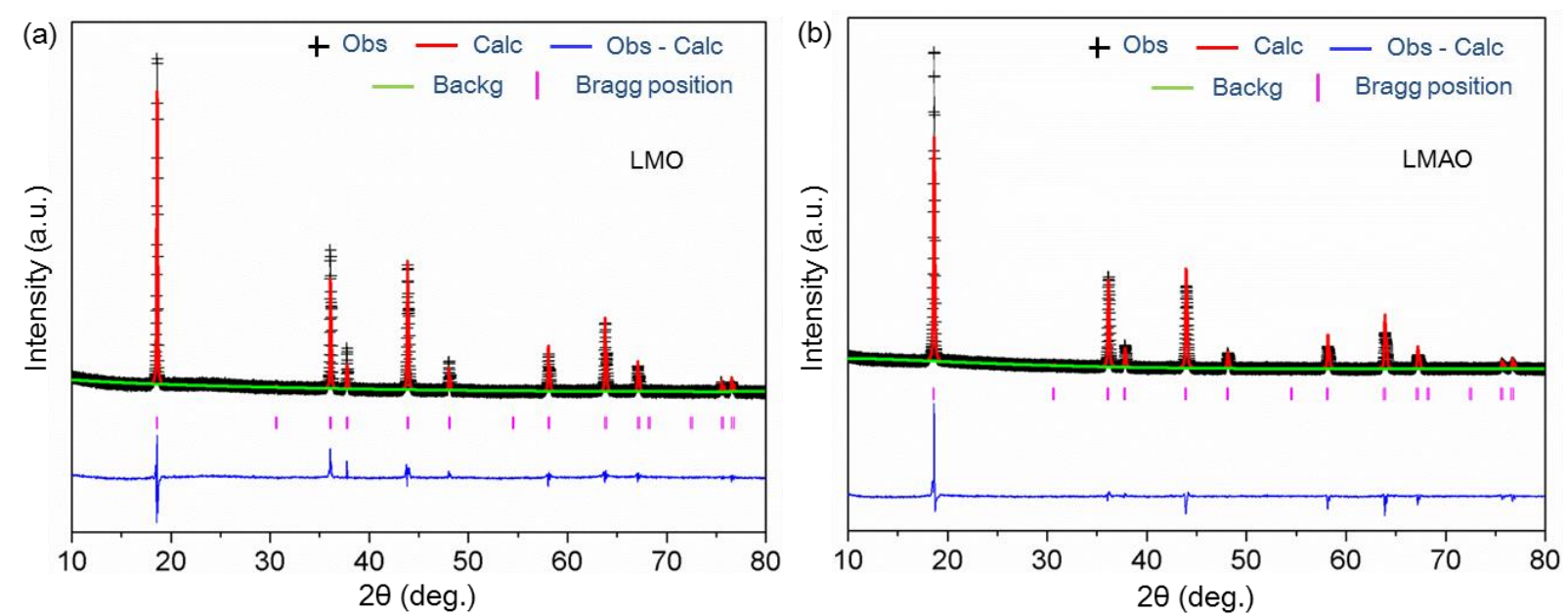

Figure S2. Rietveld refinement data from X-ray diffraction patterns of (a) LMO and (b) LMAO. 


\begin{tabular}{cccc}
\hline \hline Compounds & Region & $\varepsilon_{i}^{f}[\mathrm{eV}]$ & $\kappa_{i}^{f}\left[\mathrm{eV} \cdot \mathrm{nm}^{2}\right]$ \\
\hline \multirow{2}{*}{ LMO } & $0.0 \sim 0.125$ & 0.0 & 0.0 \\
& $0.125 \sim 0.375$ & 44.7386 & 5.7696 \\
& $0.375 \sim 1.0$ & 4.0689 & 0.5247 \\
\hline & $0.0 \sim 0.25$ & 0.0 & 0.0 \\
LMAO & $0.25 \sim 0.625$ & 14.2982 & 1.8538 \\
& $0.625 \sim 0.875$ & 31.0502 & 4.0257 \\
& $0.875 \sim 1.0$ & 0.0 & 0.0 \\
\hline \hline
\end{tabular}

Table S1. First-principles bulk free energy coefficient, $\varepsilon_{i}^{f}$, and the corresponding gradient energy coefficient, $\kappa_{i}^{f}$, at various reaction regions in LMO and LMAO. 